\title{
INDIVIDUAL- AND COMMUNITY-LEVEL DETERMINANTS OF ANTENATAL HIV TESTING IN ZIMBABWE
}

\author{
MARTIN MARUFU GAZIMBI ${ }^{1}$ AND MONICA AKINYI MAGADI \\ School of Education and Social Sciences, University of Hull, UK
}

${ }^{1}$ Corresponding author. Email: m.gazimbi@2013.hull.ac.uk

Summary. This study contributes to the dialogue on the prevention of mother-to-child HIV transmission (PMTCT) through the use of HIV and antenatal care (ANC) integrated services. The determinants of antenatal HIV testing in Zimbabwe were explored. Multilevel logistic regression models were applied to data for 8471 women from 406 clusters who gave birth in the 5 years preceding Zimbabwe Demographic and Health Surveys conducted in 2005/6 and 2010/11. The uptake of antenatal HIV testing was found to be determined by a wide range of individual-level factors relating to women's economic and demographic status, as well as HIV-related factors, including HIV awareness and stigma within the community. Important individual-level enabling and perceived need factors included high socioeconomic status, not having observed HIV-related stigma and knowledge of HIV status (based on a previous HIV test), such that these groups of individuals had a significantly higher likelihood of being tested for HIV during pregnancy than their counterparts of lower socioeconomic status, and who had observed HIV-related stigma or did not know their HIV status. The results further revealed that community HIV awareness is important for improving antenatal HIV testing, while stigma is associated with reduced testing uptake. Most contextual community-level factors were not found to have much effect on the uptake of antenatal HIV testing. Therefore, policies should focus on individual-level predisposing and enabling factors to improve the uptake of antenatal HIV testing in Zimbabwe.

\section{Introduction}

The persistent high rates of paediatric HIV transmission in sub-Saharan African countries have prompted calls for a scaling-up of access to antenatal health care services in order to reduce mother-to-child HIV transmission (MTCT). This has culminated in MTCP prevention programmes (PMTCT) being integrated with antenatal care (ANC) services as a means of reaching out to pregnant women who are HIV positive and those who are vulnerable to HIV infection. The rationale for the integration of these services is to increase the coverage of PMTCT services as pregnant women who use ANC services can also benefit from PMTCT services, and vice versa (Obure et al., 2016). It is acknowledged that prevention of parent-to-child HIV transmission (PPTCT) is the currently preferred term, but PMTCT is used in this research to reflect the fact that antenatal HIV testing services do not extend to include women's partners in Zimbabwe. Thus, the focus of this paper is on the HIV testing of mothers during antenatal care visits, rather than both parents.

There has been widespread international recognition that the goal of PMTCT cannot be met without increasing antenatal HIV testing uptake (Granich et al., 2015). World Health Organization guidelines state that routine antenatal HIV testing should be offered to all pregnant women during their first contact with maternal health care services (WHO, 2015). This is particularly crucial in high HIV prevalence settings of sub-Saharan Africa such as Zimbabwe. While the rationale for incorporating HIV testing into ANC services is strong in order to increase the uptake of antenatal HIV testing (WHO, 2015), it has been documented that even when HIV testing is offered as a part of antenatal care, many pregnant women in Zimbabwe are still only tested for the first time when they go into labour, putting their infants at risk of HIV MTCT (Heemelaar et al., 2014; Maruva et al., 2014; Buzdugan et al., 2015). Although the 2010/11 Zimbabwe Demographic and Health Survey (ZDHS) reported that $90 \%$ of pregnant women in Zimbabwe had at least one ANC visit, only 59\% received counselling and accepted an HIV test as part of their antenatal care. The level of antenatal HIV testing remains far below the $90 \%$ target adopted in the country (WHO, 2015; ZNASP, 2015-2018). In order to improve the efficacy of the PMTCT programme in Zimbabwe, it is imperative that the factors responsible for the low uptake of antenatal HIV testing are identified.

It is difficult to quantitatively measure the efficacy of integrated services, but stand-alone studies have been able to shed light on the determinants of antenatal HIV testing uptake in sub-Saharan African countries (Chandisarewa et al., 2007; Asefa \& Beyene, 2013; Heemelaar et al., 2014; Jayleen et al., 2016). The determinants 
of antenatal HIV testing may be classified into community- and individual-level predisposing, enabling and perceived risk/need factors. Low antenatal HIV testing uptake has been linked to individual-level predisposing factors such as place of residence, education, age, marital status, unwanted pregnancy, religion and parity (Ahmed et al., 2010; Jayleen et al., 2016; Ochako et al., 2016). It has been noted that place of residence shows a particularly strong association with health service utilization. Pregnant women living in urban areas have been shown to be more likely to have antenatal HIV testing than those from rural areas (Titaley et al., 2010; Munjoma et al., 2010; Semali et al., 2014; Rutaremwa \& Kabagenyi, 2016) due to easier accessibility of health care services (Allam et al., 2015).

Besides place of residence, a demographic predisposing factor that has been noted to show a particularly strong association with antenatal HIV testing is maternal age. Older women have been demonstrated to be more likely to have an antenatal HIV test than younger women (Jayleen et al., 2016; Rutaremwa \& Kabagenyi, 2016). This has been attributed to older women having a greater awareness of the dangers associated with HIV/AIDS (Byamugisha et al., 2010; Asefa \& Beyene, 2013; Birhane et al., 2015; Sama et al., 2017) or better resources, enabling them to access maternal health care services (Aremu et al., 2011; Tarekegn et al., 2014). A critical issue surrounding access to antenatal HIV testing for pregnant teenage women in sub-Saharan African countries is pregnancy wantedness and acceptance by the community (Magadi et al., 2007). Studies in Zambia and Uganda have shown that pregnant teenagers are more likely to experience violence from parents, expelled from school and to experience stigma (Denison et al., 2008; Rutaremwa and Kabagenyi, 2016). The fact that ANC services in sub-Saharan African countries are not often oriented to the needs of teenage mothers makes the health risks associated with their pregnancies and HIV more pronounced than those of older women (Matthews et al., 2009; Renju et al., 2010). There is on-going debate on the relative importance of parity as opposed to maternal age in access to HIV and ANC services. However, it has been shown that higher-order parity is associated with increased uptake of antenatal HIV testing (Tweya et al., 2014).

The marital status of pregnant women is also an important predisposing factor for both HIV testing and use of ANC services. Heemelaar et al. (2014) noted that married women are more likely to be HIV tested during pregnancy if they make more decisions in the household, if they are educated and if they regularly watch television or read newspapers. Unlike single women (i.e. the unmarried, divorced or widowed), married women may have a lower perceived risk of acquiring HIV infection (assuming the partners are mutually faithful) - a potential barrier to HIV testing (Keogh et al., 2009; Mapuranga et al., 2015).

A number of enabling factors, including socioeconomic factors (e.g. wealth, education, media exposure and physical access to health facility), HIV/AIDS factors (e.g. HIV awareness, HIV stigma) and perceived risk and need factors (e.g. history of HIV testing, acquaintance with a person living with HIV) have been linked to antenatal HIV testing in sub-Saharan African countries (Ahmed et al., 2010; Gustav et al., 2014; Jayleen et al., 2016). There is evidence in the literature that pregnant women from wealthier households are more likely to have antenatal HIV testing than their counterparts from poorer households. This may be due to women from wealthier households having the ability to afford the cost of health care services and being more likely to be educated, and women who are educated being more likely to have access to information and enjoy greater freedom to seek health care without seeking the permission of their husbands (Houweling et al., 2007; Fotso et al., 2009; Ahmed et al., 2010). It has also been a consistent finding that those with the highest levels of educational attainment are more likely to be tested for HIV during pregnancy than those at the lower end of the education spectrum (Killam et al., 2010; Sahlu et al., 2014), partly due to better access to mass media (Sama et al., 2017). Several authors have documented the role of mass media in disseminating information on health-related issues through radio, television, newspapers and magazines (Jesmin et al., 2013; Gustav et al., 2014). In recent years, HIV prevention and sexual and reproduction health promotion information have been disseminated through posters, mobile phones and the internet (Underwood \& Schwand, 2014) and this intervention has explicitly tended to increase knowledge relating to mother-to-child HIV transmission and PMTCT programmes.

Although the association between individual demographic characteristics, socioeconomic status and cultural practices is well understood in Zimbabwe and elsewhere in sub-Saharan Africa (Kevany et al., 2012; Jesmin et al., 2013), this is not true of the possible impact of HIV/AIDs enabling/disabling factors on HIV and ANC integrated services. Due to the stigma attached to HIV/AIDS, pregnant women living with HIV and those who perceive themselves to be at risk of HIV infection may be less likely to have antenatal HIV testing within the integrated services. The studies of Turan et al. (2008) and Angotti et al. (2009) suggested that the requirement of antenatal HIV testing when seeking ANC services may increase the potential for involuntary and unwanted disclosure of HIV status. It has been found that involuntary and unwanted disclosure of HIV status may heighten fears of experiencing stigma and discrimination, not only from health care providers, but also from friends, family and the community after diagnosis (Turan et al., 2008). Multiple studies in various countries have reported that perceived lack of confidentiality decreases the likelihood of HIV and ANC service utilization (Angotti et al., 2009; Mucheto et al., 2011; Hardon et al., 2012; Sahlu et al., 2014). This is because, in most sub-Saharan Africa countries, hospital records and information are recorded in hand-held notes, and these may be seen by friends and family members, thereby inadvertently discouraging pregnant women from utilizing HIV and ANC integrated services (Demissie et al., 2009; Washington et al., 2015).

Personal perceived risk of HIV infection and the need to seek health care as a protective measure against HIV have also been found to be associated with an increased likelihood of HIV testing and use of ANC services (Lepine $e t$ 
al., 2014). Depending on past sexual behaviour, HIV awareness and personal acquaintance with people infected with HIV/AIDS, individuals construct their own perceived probability of being infected, affecting their health-seeking behaviour. Being personally affected by HIV, especially seeing a close person die of HIV, may be associated with a higher perceived risk of HIV infection, and perception of personal vulnerability leads to the adoption of protective behaviour (Angotti et al., 2009; Sahlu et al., 2014). Therefore, being HIV positive or knowing someone living with $\mathrm{HIV}$ is expected to influence the perceived need to access HIV and ANC integrated services as a protective measure against MTCT (Maruva et al., 2014).

The bulk of studies on maternal health care in Zimbabwe have largely focused on individual-level demographic and socioeconomic factors (Muchabaiwa et al., 2012; Maruva et al., 2014; Makate \& Makate, 2017), but the role of HIV/AIDS was not considered in these studies. Only one study, Maruva et al. (2014), made reference to the effect of knowledge of HIV status on maternal health service utilization, and this supported the claim that maternal health uptake has been decreased by the requirement for HIV testing during pregnancy. While studies in Zimbabwe may shed light on the determinants of HIV and ANC service utilization, the overall drawback of these has been an apparent failure to separate community-level factors from individual-level variables. The importance of contextual community factors has been established by other studies (Magadi et al., 2007; Alam et al., 2015; Chitalu \& Koch, 2015), explaining the significant cluster variations that remain after controlling for the effect of individual-level variables. The context within which individuals live matters because the broader environment not only provides opportunities to learn and reassure, but also generates general norms regarding activities within the community (O'Meara et al., 2013). For example, due to observed stigma and discrimination against people living with HIV in communities, women living with HIV, and those who perceive themselves to be at risk of HIV infection, may be less likely to use integrated reproductive health care services (Sharma et al., 2017). This study therefore aimed to address this evidence gap by implementing a multilevel modelling approach (Goldstein, 2003) to explicitly examine both the individual- and contextual-level determinants of using integrated HIV and ANC services. Understanding and addressing individual and community barriers to antenatal HIV testing could potentially contribute to the current goal of eliminating the MTCT of HIV in Zimbabwe.

\section{Conceptual framework}

In order to examine the community- and individual-level determinants of antenatal HIV testing, the conceptual framework of Andersen (1995) was adopted. Figure 1 provides a schematic display of this framework. The model was conceptualized based on predisposition, enablement and need for health care use. The predisposing factors (individual and community level) were assumed to operate through enabling and perceived need factors. The enabling factors included the social and economic status of a pregnant women (i.e. household wealth, access to media, education, women's autonomy) and HIV enabling or disabling factors (i.e. HIV awareness, stigma and confidentiality concern). While enabling factors are assumed to facilitate access to HIV and ANC services, pregnant women must perceive themselves to be at risk and believe the seriousness of HIV infection to induce the need to access HIV and ANC services (Iriyana, 2007). For example, witnessing the death or illness of a close friend or a family member due to HIV may compel pregnant women to have antenatal HIV testing if they know the effectiveness of PMTCT programmes in reducing MTCT (Catania, 1990). For that reason, the perceived need for antenatal HIV testing or whether pregnant women knew someone living with HIV were also considered in the framework. The framework also recognizes that community variables have independent effects on health care services and may also influence enabling and need factors. For example, individuals resident in communities with higher HIV prevalence may be more likely to perceive themselves to be at risk of HIV infection, and perceived risk of HIV infection is a major determinant of HIV testing (Turan et al., 2008).

In this study, community-level factors included HIV prevalence, stigma and awareness in the community.

\section{Methods}

\section{Data}

The study was based on data from the 2005/6 and 2010/11 Zimbabwe Demographic and Health Surveys (ZDHS). A total of 18,078 women were interviewed in the two surveys. To construct the sample for analysis from merged ZDHS datasets, the selection was initially restricted to all women who had given birth during the 5 years preceding the surveys (DHS code; V208>0). Thus women who did not give birth within the specified period were excluded, generating an unweighted sample of 8471 women who gave birth to a total of 10,620 children. To avoid clustering of multiple births per women and to reduce the likelihood of recall bias on sensitive questions (Uthman $e t$ al., 2017), the sample was further limited to the last birth in the 5 years preceding the surveys (DHS code; BIDX $\$ 01=1)$. This restriction gave a final sample of 8471 women with at least one live birth in the 5 years preceding the surveys. Of these, 92.4\% $(n=7879)$ had at least one ANC visit, whilst 7.6\% $(n=648)$ did not, representing near-universal access to ANC services. From this sample, dependent and independent variables of interest were extracted. 


\section{HIV testing during ANC}

The key outcome variable for the study was whether the respondent had antenatal HIV testing during the last pregnancy. In the ZDHS, pregnant women who attended ANC services for the most recent birth were asked the following questions: (a) Were you tested for the AIDS virus during any of the ANC visits? If yes, (b) Did you get the results of the AIDS test? Pregnant women have to get tested and know their HIV status before they can be enrolled in a PMTCT programme, so women who answered 'yes' to these two questions were classified as having been tested for HIV during the ANC visit. Those who answered 'no' to both questions were considered not to have been tested for HIV during the ANC visit (Staveteg et al., 2013). The measure was based on the DHS code V840-1. Women who were tested for HIV were coded ' 1 ' and those who were not were coded ' 0 ', i.e. this was a dichotomous response variable.

\section{Explanatory variables}

Based on the literature and the conceptual framework, a number of predisposing factors were selected as explanatory variables, including individual demographic factors (i.e. age, marital status, religion, place of residence, year of survey, parity, wanted child) and community HIV factors that predispose pregnant women to using integrated services (i.e. HIV awareness, stigma and prevalence). Enabling factors included the logistic and resource aspects of obtaining care, which comprised household income, access to media, and availability and accessibility of health care facilities. The HIV and psychological enabling factors included HIV stigma, awareness, disclosure and confidential concern. The need factors related to the perceived and evaluated assessment of an individual's own health status, which may inform the need to seek health care (Lepine et al., 2014). In line with other studies, 'ever been tested for HIV' (a proxy for knowledge of HIV status) and 'knowing someone living with HIV' were considered as proxies for the perceived risk and need factors that may have influenced the health-seeking behaviour of the women. Community-level HIV awareness, stigma and media exposure variables were imputed from relevant individual-level factors. A description of the individual-level and community-level variables included in the analysis is given in Table 1.

\section{Analysis}

Multilevel binary logistic regression analysis was used, with community at Level 2 and last birth in the last 5 years at Level 1. The logit of the probability of an outcome can be modelled as follows:

$$
\begin{gathered}
f\left(\pi_{i j}\right)=\log \log \left[\frac{\pi_{i j}}{1 \pi_{i j}}\right]=\beta_{0}+\beta_{1} X_{1 i j}+\beta_{2} C_{2 i j}+\mu_{0 j} \\
\operatorname{Var}\left(u_{0 j}\right)=\sigma_{u 0}^{2}
\end{gathered}
$$

where $\pi_{i j}$ is the probability of an outcome for a pregnant women, $i$, in the $j^{\text {th }}$ community. The intercept $\beta_{0}$ is shared by all communities; $\beta$ values denote fixed coefficients; $X$ and $C$ are vectors of covariates at individual and community levels, respectively; and $\mu_{0 j} \sim N\left(0, \sigma^{2} j\right)$ shows the random effects for the $j^{\text {th }}$ community. The random intercept is shared by all individuals from the same community (defined by a primary sampling unit or cluster) and serves as an indirect control for community factors not included in the models that may affect antenatal HIV testing.

Before including any explanatory variables in the models, it was crucial to know how much between-community variation there was in the propensity for antenatal HIV testing. To assess this, the estimated value of $\sigma_{u 0}^{2}$-the variance of $u_{0 j}$ - was examined. A threshold model approach was used, which measures the intra-cluster correlation (ICC) as:

$$
\text { ICC }=\sigma_{u}^{2} /\left(\sigma_{u}^{2}+\sigma_{\varepsilon}^{2}\right)
$$

where $\sigma_{u}^{2}$ is the total variance at the community level, and $\sigma_{\varepsilon}^{2}$ is the total variance at individual level. For the multilevel logistic regression model, the Level 1 residuals were assumed to have a standard logistic distribution with mean zero and variance $\sigma_{\varepsilon}^{2}=\pi^{2} / 3$, where $\pi$ is the constant 3.14... Thus ICC $=\sigma_{u}^{2} / \sigma_{u}^{2}+3.29$ (Goldstein, 2003).

The proportional change in variance (PCV) was derived from:

$$
P C V=V_{A} V_{B} \times 100
$$

where: $V_{\mathrm{A}}=$ variance of the initial model and $V_{\mathrm{B}}=$ variance of the succeeding model.

The deviance information criterion (DIC) was used as a measure of the goodness-of-fit of the models. A lower DIC value in subsequent models indicates a better fit of the model (Aremu et al., 2011).

Multilevel logistic regression analysis was used to determine the factors that may have influenced antenatal HIV testing. This was performed using MLwiN statistical software. The first model (Model 0) was an intercept-only model (a so-called empty model) with year of survey as the only covariate. This empty model was useful to show if there was sufficient variance at the community level. All the individual predisposing factors were added simultaneously in Model 1. In Model 2, enabling and perceived need factors were added and adjusted for. The community-level factors were then included in Model 3. All the fixed effects were expressed as average odds ratios (AORs) in the multilevel models in Table 5, presented with 95\% confidence intervals ( $95 \% \mathrm{CIs}$ ). The random effects are expressed as variance partition coefficients (VPCs), also known as intra-class correlation coefficients (ICCs), and 


\section{Results}

\section{Bivariate analysis}

The characteristics of the sample women are presented in Table 2, and the bivariate distributions of the women by whether they had had an antenatal HIV test and by different predisposing, and enabling and need, factors are presented in Tables 3 and 4, respectively. The bivariate analysis results generally indicate that the delivery of integrated HIV and ANC health services in Zimbabwe over recent years has resulted in a substantial increase in antenatal HIV testing uptake. The uptake of antenatal HIV testing increased dramatically between the two survey periods, from $29.4 \%$ in $2005 / 6$ to $55.9 \%$ in $2010 / 11$ (a percentage point difference of $26.5 \%$ ). The most dramatic increases in antenatal HIV testing uptake were found among women resident in rural areas, those under the age of 35 years, never-married and married women, those with secondary or higher level educational attainment, those of higher order parity and those with low to medium exposure to media. For example, antenatal HIV testing uptake increased dramatically from $24.4 \%$ in $2005 / 6$ to $54.9 \%$ in $2010 / 11$ among rural women (Table 3 ). It also increased significantly among pregnant women with medium to higher HIV awareness, women who knew someone living with HIV and among those who reported having no problem accessing a health facility. An increase in antenatal HIV testing was also observed across all wealth categories, with the rich-poor gap narrowing (Table 4).

\section{Multilevel logistic regression analysis}

Table 5 shows the results of the multilevel logistic regression analysis. The empty model (Model 0) for antenatal HIV testing (not presented in table) suggested that most of the variation in antenatal HIV testing was at the individual level. However, there was also some variation at the community level, as indicated by the significant variance. Based on the estimated VPC, the intra-community correlation (ICC) was estimated at about $8 \%$. This indicated the extent of the variability in antenatal HIV testing that could be attributed to community-level factors. The likelihood of having antenatal HIV testing was 3.77 times higher $(A O R=3.77,95 \% \mathrm{CI}=3.32-4.29)$ in the 2010/11 ZDHS than in the 2005/6 ZDHS.

All significant predisposing individual-level factors were included in Model 1. The variables found to be associated with antenatal HIV testing in Model 1 included place of residence, age group, marital status, women's educational attainment and parity. While urban residents may be expected to have better access to health care facilities in general, the results revealed that pregnant women from urban areas of Zimbabwe were $48 \%$ less likely $(\mathrm{AOR}=0.52,95 \% \mathrm{CI}=0.43-0.63)$ to be tested for HIV than their rural counterparts of similar background socioeconomic and demographic characteristics. The results also showed that age of women, being previously married (divorced or widowed) and parity were inversely correlated with antenatal HIV testing. The estimates suggested antenatal HIV testing decreased with age, such that women of age groups 25-29, 30-34 and over 35 years were less likely to have been tested for HIV during ANC visits by $29 \%$ (AOR=0.71, 95\% CI $=0.62-0.81$ ), 31\% $(\mathrm{AOR}=0.69,95 \% \mathrm{CI}=0.56-0.70)$ and $57 \% \quad(\mathrm{AOR}=0.43,95 \% \mathrm{CI}=0.36-0.52)$, respectively, compared with younger women in the age group 15-24. Within marital status categories, divorced women were $26 \%$ less likely to have an antenatal $\mathrm{HIV}$ test $(\mathrm{AOR}=0.74,95 \% \mathrm{CI}=0.57-0.96)$ compared with never-married pregnant women with similar background characteristics. Other marital status categories, i.e. being currently married or widowed, were not found to be significantly different from never-married women with respect to antenatal HIV testing. Women with a second-order birth were $28 \%$ less likely ( $\mathrm{AOR}=0.72,95 \% \mathrm{CI}=0.58-0.90)$ to have antenatal HIV testing than those with first-order births. Having secondary or higher educational attainment was found to be associated with an increased odds of having an antenatal HIV test by 1.43 times $(\mathrm{OR}=1.43,95 \% \mathrm{CI}=1.28-1.61)$, compared with women with primary or no education. There was no evidence of an association between religious affiliation and wantedness of a child with antenatal HIV testing in Model 1.

In Model 2, enabling and HIV need factors were added to Model 1. When these factors were controlled for, most patterns for predisposing factors remained unchanged, but the association between place of residence and antenatal HIV testing ceased to be significant. Being wealthier was found to be associated with increased odds of antenatal HIV testing. The estimates suggest that women in the richer and richest household quintiles were 1.45 $(\mathrm{AOR}=1.45,95 \% \mathrm{CI}=1.11-1.91)$ and $1.67(\mathrm{AOR}=1.67,95 \% \mathrm{CI}=1.16-2.41)$ times more likely to have had an antenatal HIV test, compared with women from poor (poorest, poorer or middle quintiles) households. This association between antenatal HIV testing with household wealth continued into Model 3, which included contextual community-level factors. The likelihood of antenatal HIV testing was also found to be significantly associated with media exposure. Pregnant women who had medium or high media exposure were more likely to have antenatal HIV testing than those with low media exposure. For example, women who had high media exposure were 1.30 times $(\mathrm{AOR}=1.30,95 \% \mathrm{CI}=1.06-1.61)$ more likely to have had an antenatal HIV test, compared with those with low media exposure. Women with higher media exposure continued to have a higher likelihood of antenatal HIV testing. even after community-level factors were controlled for. The odds of having an HIV test during an ANC visit were on 
average $14 \%$ lower among women who reported having problems in accessing health care centres in Model 2 and 3 , compared with those who said access was not a major problem. The effect of religion and child wantedness turned significant in Models 2 and 3. Apostolic women were more likely to have had antenatal HIV testing than Roman Catholic women. On the other hand, women who said they wanted a child later were less likely to have had an antenatal HIV test than those who reported wanting a child then. There was no evidence that women's decision-making/autonomy in the household or parity had a significant effect on antenatal HIV testing when other considered variables were controlled for in the model.

Model 2 also considered HIV-related factors, i.e. being previously tested for HIV (a proxy for knowledge of HIV status), awareness and confidentiality concerns. These were not found to be significantly associated with antenatal HIV testing across the models. However, knowing someone living with HIV was associated with an increased odds of antenatal HIV testing. Women who knew someone living with HIV were on average 1.24 times more likely to have had an antenatal HIV test compared with their counterparts who knew no one with HIV.

Community-level variables included in Model 3 were HIV stigma, HIV awareness and HIV prevalence. The fixed effects from Model 3 were about the same as with Models 1 and 2. There was no evidence of a significant association between HIV prevalence at the community level and antenatal HIV testing. However, women residing in communities with high HIV awareness were 2.08 times $(2.08,95 \% \mathrm{CI}=1.05-4.11)$ more likely to have been tested for HIV compared with their peers who lived in communities with low HIV awareness. The results also showed that living in a community with a high proportion of individuals with stigma was associated with reduced odds of antenatal HIV testing. Compared with Model 2, the final ICC remained at 7\%, with zero proportional change in variance being observed. The successive reduction in DIC from Models 0 to 3 demonstrates a better fitting model over every previous one.

\section{Discussion}

The main objective of this study was to provide an overall picture of the individual- and community-level determinants of antenatal HIV testing in Zimbabwe. Some correlation was found among individuals from the same community (Aremu et al., 2011). This was as expected, given the different tribal, cultural and religious contexts in Zimbabwe, which to some extent serve as markers of attitudinal identities, socioeconomic status and health beliefs towards health care service utilization (Kayeyi et al., 2009). After controlling for the study's selected variables, the intra-community correlation for antenatal HIV testing was $7 \%$, suggesting that over $90 \%$ of the total unexplained variation in antenatal HIV testing was due to unobserved individual-level factors.

The study found that antenatal HIV testing had increased significantly between 2005/6 and 2010/11 in Zimbabwe. Individual predisposing and enabling socioeconomic status factors appeared to be more important than community-level factors for antenatal HIV testing within integrated HIV/ANC services in the country. Most of the factors that were found to be associated with antenatal HIV testing were largely consistent with patterns observed in other sub-Saharan African countries (e.g. place of residence, age, marital status, education, parity, child wantedness and religion). The observed patterns for place of residence contradicted the results of earlier studies, which found that women from urban areas were more likely to have antenatal HIV testing due to easier access to ANC services, including PMTCT (Martin-Herz et al., 2006; Mucheto et al., 2011; Maruva et al., 2014). The lack of significance in the association between place of residence and antenatal HIV testing when key factors were controlled for suggests that PMTCT programmes in Zimbabwe are now achieving comparable HIV testing coverage in urban and rural areas.

The finding that older women were less likely to have antenatal HIV testing was not consistent with the findings of previous studies in other settings, which found that antenatal HIV testing increased with women's age (Koech et al., 2014; Tweya et al., 2014). Previous studies have suggested that older women have higher odds of accepting antenatal HIV testing due to their accumulated knowledge of HIV, especially that HIV can be transmitted to a child during pregnancy, as well as their knowledge of the existence of PMTCT programmes (Semali et al., 2014; Woldesenbet et al., 2015). A possible explanation for this could be that older women may have previously given birth to presumably healthy children, and thus see no reason to be HIV tested in future pregnancies. They may also have had a perceived low risk of HIV infection if they were married, assuming their partners were faithful (Byamugisha et al., 2010). It may also be that older women have already witnessed discrimination against, and isolation from, HIV-positive women (Rutaremwa \& Kabagenyi, 2016), and having an HIV diagnosis during pregnancy may increase their sense of discrimination and isolation. These feelings of discrimination, isolation and low perception of risk of HIV infection could be overcome through effective communication and campaigns to reduce stigma and increase knowledge of MTCT among older pregnant women (Mtumbuka et al., 2012). There is a need to develop new methods of communication and tools targeted at older women (Maruva et al., 2014).

On the other hand, the study results show that young adults are more likely to engage in high-risk sexual behavior, including having multiple sexual partners, and therefore may have a higher perceived HIV risk, encouraging them to seek an HIV test when they get pregnant (Musarandega et al., 2017). In addition, health care service providers may believe that young adults are at a greater risk of HIV infection, and therefore be more likely to recommend an HIV test to young women than to older women (Mapuranga et al., 2015). Young pregnant women have been shown to be more likely to accept an antenatal HIV test when it is offered in an appropriate and supportive 
environment (Koech et al., 2014). Youth-friendly HIV testing centres, such as Surestart Health Centres, are improving antenatal HIV testing uptake in Zimbabwe (Musarandega et al., 2017). The study also found that antenatal HIV testing was lower among previously married women (widowed or divorced) than among never-married or married women. This might reflect the fact that ANC services in Zimbabwe are geared towards married women (Chevo \& Bhatasara, 2012). Like young pregnant women, widowed and divorced women are more likely to be in multiple sexual partnerships, increasing their perceived risk of HIV infection, thus discouraging them from accepting antenatal HIV testing for fear of discrimination and isolation if they are diagnosed with HIV (Murire et al., 2014). It is therefore important to provide information on, and to raise awareness of, PMTCT programmes among previously married women in Zimbabwe (Buzdugan et al., 2015).

Various enabling and perceived need factors were found to be major determinants of HIV and ANC integrated health service use in Zimbabwe. In particular, household wealth was found to be associated with the likelihood of antenatal HIV testing. Previous studies have shown that being wealthier is associated with an increased likelihood of using maternal health care services (Babalola \& Fatuisi 2009; Tarekegn et al., 2014). This is as expected, since women from wealthier households are more likely to be able to afford health care services and their associated costs, such as transport (Semali et al., 2014; Lepine et al., 2014; Moindi et al., 2015). Another possible explanation is that, since higher household wealth could be an indication of a higher number of educated household members, women from such households may have greater access to information and education on HIV and its implications (Chandisarewa et al., 2007; Ahmed et al., 2010).

The finding that educational attainment was a key factor for antenatal HIV testing supports the notion that educated women are more likely to realise the benefits of antenatal HIV testing (Tenthan et al., 2015). Multiple potential pathways that can explain why maternal education is associated with antenatal HIV testing have been described, including an increased knowledge of the benefits of preventative health care and awareness of risk (e.g. PMTCT), higher receptivity to new health care information and socialization with formal services outside the home, access to financial resources, better communication with partners and increased self-worth and confidence (Byamugisha et al., 2010; Asefa \& Beyene, 2013; Maruva et al., 2014; Birhane et al., 2015; Sama et al., 2017).

The study results showed mixed patterns regarding the association between enabling and perceived risk/need factors and HIV/AIDS. No evidence was found of a significant association between HIV awareness and HIV sero-status with the likelihood of antenatal HIV testing, once important background socioeconomic, demographic and other HIV/AIDS factors were controlled for. However, there was evidence of an association between HIV stigma and antenatal HIV testing. The finding that pregnant women with medium-to-high levels of stigma were more likely to have antenatal HIV testing was unexpected. The logical explanation for this is pregnant women's determination to protect their infants from HIV, notwithstanding their own experience of stigma (Mapuranga et al., 2015). The fact that antenatal HIV testing is not associated with confidentiality concern may suggest that women are given private individual counselling and space to make a private decision without staff or group interference in Zimbabwe (Chevo et al., 2012). Shetty et al. (2010) suggested that quality of care, including confidential concern, shapes a woman's decision to use health care services. Knowing someone living with HIV was found to be associated with an increased likelihood of antenatal HIV testing. The concept of the health belief model could explain this association. Witnessing someone living with HIV or dying from AIDS might lead pregnant women to seek an antenatal HIV test (Iriyana, 2007). This may suggest that personal acquaintance with people living with HIV may be a pointer to behavioural change to avoid MTCT, and knowing someone living with HIV is common in communities with a high HIV prevalence, such as in Zimbabwe (Magadi \& Desta, 2011). The finding that higher HIV awareness at the community level is associated with an increased odds of antenatal HIV testing resonates with the findings of Buzdugan et al. (2015), and is suggestive of a strong and widespread campaign of PMTCT programmes (Ngome \& Odimegwu, 2014).

There are some limitations to the study that have potential implications for interpretation of the findings. First, the 2005/6 and 2010/11 DHS data are relatively old and might not reflect the current situation and practices. In Zimbabwe, where there is a current scaling-up of PMTCT programmes, use of the 2015/16 DHS data would have helped capture more recent progress. However, these were not available at the time the study. The second limitation relates to the cross-sectional nature of the DHS data, which does not allow temporal ordering of the events of interests. Therefore, the patterns observed represent simple associations and cannot offer insight into cause and effect relationships. Furthermore, because DHS surveys rely on self-reported data collection, inaccuracies can arise as a result of intentional deception, poor memory or the misunderstanding of questions. Finally, the study focused on the major determinants of antenatal HIV testing in Zimbabwe with respect to demand-related factors (i.e. cultural, demographic and socioeconomic). However, as argued by Buzdugan et al. (2015), service-related factors are also important determinants of exclusion and need investigation. Future research should explore the impact of provider characteristics on HIV and ANC integrated service delivery. Factors such as quality of care, type of provider, actual distance and attitude of staff need to be explored. Nevertheless, the representation of the whole country is a major strength of the data analysed in this study as it allows generalization of the findings nationally. 
Overall, this study found little variation in antenatal HIV testing across communities in Zimbabwe, and any variations were mainly explained by individual-level variables such as background characteristics and enabling/perceived need factors. Household wealth, access to media and HIV factors such as knowing someone living with HIV were found to be critical for antenatal HIV testing. At the community level, contextual variables found to be contributing to the variations in HIV testing included HIV stigma and awareness. Controlling for community-level factors did not have much effect on community variations in HIV testing. Therefore, while recognizing the importance of some community-level factors, policies should focus on individual-level predisposing and enabling factors in order to improve HIV and ANC integrated services in Zimbabwe.

A number of policies can be recommended from the findings. First, the introduction of financial incentives may stimulate access to HIV and ANC integrated services by providing compensation for transport costs and opportunity costs, i.e. the time associated with accessing services. Monetary incentives could reduce socioeconomic barriers and HIV-related stigma as they may provide a broader reason for going to a health facility for antenatal care. More importantly perhaps, awareness creation on antenatal HIV testing should focus on avoiding stigmatizing and discriminatory behaviours, so that pregnant women can develop positive attitudes towards people living with HIV/AIDS. Lastly, policymakers should use electronic and mass media to communicate and spread awareness, taking advantage of the widespread access to electronic media such as mobile phones and radio in Zimbabwe. These could help fill the void created by a lack of formal education in some sections of the population. This method could also be used to influence decisions in cases where a pregnancy is mistimed or unwanted, and also change attitudes in the case of socio-cultural enabling factors such as religious affiliation.

\section{Acknowledgments}

The study data were provided by the Demographic and Health Survey (DHS) programme, ICF Macro, Calverton, MD, USA. This is authorized to distribute, at no cost, unrestricted survey data files for legitimate academic research, following registration to enable data access (http://www.dhsprogram.com/data/Using-DataSets-forAnalysis).

Ethical Approval. The research presented in this paper is based on secondary analysis of data from Zimbabwe Demographic and Health Surveys (ZDHS), which included already anonymized survey data, so ethical approval was not required.

Conflicts of Interest. The authors declare that they have no conflicts of interest.

Funding. This research received no specific grant from any agency, commercial entity or not-for-profit organisation.

\section{References}

Ahmed, S., Creanga, A. A., Gillespie, D. G. \& Tsui, A. O. (2010) Economic status, education and empowerment: implications for maternal health service utilization in developing countries. PLoS One 5, 6.

Alam, N., Hajizadeh, M., Dumont, A. \& Fournier, P. (2015) Inequalities in maternal health care utilization in sub-Saharan African countries: a multiyear and multi-country analysis. PLoS One 10, 4.

Andersen, R. M. (1995) Revisiting the behavioural model and access to medical care: does it matter? Journal of Health and Social Behaviour 36, 1-10.

Angotti, N., Bula, A., Gaydosh, L., Kimch, E. Z., Thornton, R. L. \& Yeatman, S. E. (2009) Increasing the acceptability of HIV counselling and testing with three C's: Convenience, Confidentiality and Credibility. Social Science \& Medicine 68(12), 2263-2270.

Aremu, O., Lawoko, S. \& Dalal, K. (2011) Neighbourhood socioeconomic disadvantage, individual wealth status and patterns of delivery care utilization in Nigeria: a multilevel discrete choice analysis. Women's Health 3, 167-174.

Asefa, A. \& Beyene, H. (2013) Awareness and knowledge on timing of mother-to-child transmission of HIV among antenatal care attending women in southern Ethiopia: a cross sectional study. Reproductive Health 10, 66.

Babalola, S. \& Fatusi, A. (2009) Determinants of use of maternal health services in Nigeria - looking beyond individual and household factors. BMC Pregnancy and Childbirth 9, 43-103

Bertrand, J. T., O’Reilly, K., Denison, J., Anhang, R., Sweat, M. (2006) Systematic review of the effectiveness of mass communication programs to change HIV/AIDS-related behaviours. Health Education Research 21(4), 567-597.

Birhane, T., Tessema. G., Alene, A. \& Dadi, A. (2015) Knowledge of pregnant women on mother-to-child transmission of HIV in Meket District, northeast Ethiopia. Journal of Pregnancy 4, 1-6

Buzdugan, R., McCoy, S. I., Webb, K., Mushavi, A., Mahomva, A., Padian, N. S. \& Cowan, F. M. (2015) Facility-based delivery in the context of Zimbabwe's HIV epidemic - missed opportunities for improving engagement with care: a community-based serosurvey. BMC Pregnancy and Childbirth 15, 338.

Byamugisha, R., Tumwine, J., Ndeezi, G., Karamagi, C. \& Tylleska, T. (2010) Attitudes to routine HIV counselling and testing, and knowledge about prevention of mother to child transmission of HIV in eastern Uganda: a cross-sectional survey among antenatal attendees. Journal of the International AIDS Society 13, 52.

Catania, J., Kegeles, S. \& Coates, T. (1990) Towards an understanding of risk behavior: an AIDS Risk Reduction Model (ARRM). Health Education Quarterly 17(1), 53-72.

Chandisarewa, W., Chibanda, L. S., Chirapa, E., Miller, A., Simoyi, M., Mahomva, A. et al. (2007) Routine offer of antenatal HIV testing ("opt-out" approach) to prevent mother-to-child transmission of HIV in urban Zimbabwe. Publication: Bulletin of the World Health Organization, 06-035188; doi: 10.2471/BLT.06.035188

Chevo, T. \& Bhatasara, S. (2012) HIV and AIDS programmes in Zimbabwe: implications for the health system. Journal of Perinatology 30(11), 763 
Chitalu, M. C. C. \& Koch, S. F. (2015) Utilisation of focused ANC in Zambia: examining individual and community level factors using a multilevel analysis. Health Policy and Planning 30(10), 78-87.

Demissie, A., Deribew, A. \& Abera, A. (2009) Determinants of acceptance of voluntary HIV testing among antenatal clinic attendees at Dil Chora Hospital, Dire Dawa, East Ethiopia. Ethiopian Journal for Health Development 23(2), 000-000.

Denison, J. A., McCauley, A. P., Dunnett-Dagg, W. A., Lungu, N. \& Sweat, M. D. (2008) The HIV testing experiences of adolescents in Ndola, Zambia: do families and friends matter? AIDS Care 20(1), 101-105.

Fotso, J. C., Ezeh, A., Madise, N., Ziraba, A. \& Ogollah, R. (2009) What does access to maternal care mean among the urban poor? Factors associated with use of appropriate maternal health services in the slum settlements of Nairobi, Kenya. Maternal Child Health Journal 13(1), 130-137.

Goldstein, H. (2003) Multilevel Statistical Models, $3^{\text {rd }}$ edition. Arnold, London.

Granich, R., Gupta, S, Hersh, B. et al. (2015) Trends in AIDS deaths, new infections and ART coverage in the top 30 countries with the highest AIDS mortality burden; 1990-2013. PLoS One, 10:e0131353.

Gustav, A. S. P., Odberg Pettersson, K., Sandberg, J., Kabakyenga, J. \& Agardh, A. (2014) Associations between mass media exposure and birth preparedness among women in southwestern Uganda: a community-based survey. Global Health Action 7(9), 1-9.

Hardon, A., Vernooij, E., Bongololo-Mbera, G., Cherutich, P., Desclaux, A., Kyaddondo, D. et al. (2012) Women's views on consent, counseling and confidentiality in PMTCT: a mixed-methods study in four African countries. BMC Public Health 12, 26.

Heemelaar, S., Habets, N., Makukuka, Z. \& Van Den Akker, T. (2014) Repeat HIV testing during pregnancy and delivery: missed opportunities in a rural district hospital in Zambia. Tropical Medicine \& International Health 20(3), 000-000.

Houweling, T. A., Ronsmans, C. \& Campbell, O. (2007) Huge poor rich inequalities in maternity care: an international comparative study of maternity and child care in developing countries. Bulletin of the World Health Organization $\mathbf{8 5}$, $745-754$.

Iriyana, S., Nakahara, S., Jimba, M. et al. (2007) AIDS health beliefs and intention for sexual severity and susceptibility. Science Direct 121(1), 64-72

Jayleen, K. L. G., Asaolu, I. O., Center, E. K., Gibson, S. C., Wightman, P., Echezona, E. \& Ehiri, J. E. (2016) Antenatal care and uptake of HIV testing among pregnant women in sub-Saharan Africa: a cross-sectional study. Journal of the International AIDS Society 19, 20605

Jesmin, S. S., Chaudhuri, S. \& Abdullah, S. (2013) Educating women for HIV prevention: does exposure to mass media make them more knowledgeable? Health Care Women International 34, 30331.

Kabagenyi, A., Reid, A., Ntozi, J. \& Atuyambe, L. (2016) Socio-cultural inhibitors to use of modern contraceptive techniques in rural Uganda: a qualitative study. Pan African Medical Journal 25, 78.

Kayeyi, N., Sanday, I. F. \& Fykesnes, K. (2014) Effects of neighbourhood-level educational attainment on HIV prevalence among young women in Zambia. BMC Public Health 9, 310.

Keogh, S. C., Urassa, M., Kumogola, Y., Mngara, J. \& Zaba, B. (2009) Reproductive behaviour and HIV status of antenatal clients in northern Tanzania: opportunities for family planning and preventing mother-to-child transmission integration. AIDS 23, S27-S35.

Kevany, S., Murimo, O., Singh, B. et al. (2012) Socio-economic status and health care utilisation in rural Zimbabwe: findings from Project Accept (HPTN 043). Journal of Public Health in Africa 3(1), e13.

Killam, W. P., Tambatamba, B. C., Chintu, N., Rouse, D., Stringer, E., Bweupe, M. et al. (2010) Antiretroviral therapy in antenatal care to increase treatment initiation in HIV-infected pregnant women: a stepped-wedge evaluation. AIDS 24(1), $85-91$.

Koech, E., Teasdeale, C. A., Wang, C. et al. (2014) Characteristics and outcomes of HIV-infected youth and young adolescents enrolled in HIV care in Kenya. AIDS 28, 2729-2738.

Lepine, A., Terris-Prestholt, F. T. \& Vickerman, P. (2014) Determinants of HIV testing among Nigerian couples: a multilevel modelling approach. Health Policy and Planning 30(5), 579-592.

Magadi, M. A., Agwanda, A. O. \& Obare, F. O. (2007) A comparative analysis of the use of maternal health services between teenagers and older mothers in sub-Saharan Africa: evidence from Demographic and Health Surveys (DHS). Social Science \& Medicine 64(6), 1311-1325.

Magadi, M. \& Desta, M. (2011) A multilevel analysis of determinants and cross-national variations of HIV sero positivity in sub-Saharan Africa: evidence from the DHS. Health and Place 17, 1067-1083

Makate, M. \& Makate, C. (2017) The evolution of socioeconomic-related inequalities in maternal healthcare utilization: evidence from Zimbabwe, 1994-2011. Global Health Research and Policy 2(1), 000-000.

Mapuranga, B., Rugonye, S. \& Pombi, S. (2015) Women's perception on HIV/AIDS testing during pregnancy a case of Mopani District in Nkowankowa Tzaneen South Africa. Journal of Health, Medicine and Nursing 19, 2422-8419.

Martin-Herz, S. P., Shetty, A. K., Bassett, M. T., Ley, C., Mhazo, M., Moyo, S. et al. (2006) Perceived risks and benefits of HIV test and predictors of acceptance of HIV counselling and testing among pregnant women in Zimbabwe. International Journal of STDS and AIDS 17(12), 835-841.

Maruva, M., Gwavuya, S., Marume, M., Musarandega, R. \& Madzingira, N. (2014) Knowledge of HIV status at ANC and utilisation of maternal health services in the 2010-11 Zimbabwe Demographic and Health Survey. Zimbabwe Working Papers No. 8. ICF International, Rockville, MD, USA.

Mashuba, S., Hemalata, K.(2015) HIV status disclosure among HIV positive women in Zimbabwe. The International Asian Research Journal 3 (01), 44.50.

Matthews, C., Guttmacher, S., Fisher, A., Mtshizana, Y., Nelson, T., McCarthy, J. \& Daries, V. (2009) The quality of HIV testing services for adolescents in Cape Town, South Africa: do adolescent-friendly services make a difference? Journal of Adolescent Health 44, 188-190. 
Moindi, R. O., Mbakaya, C., Nyambati, V. \& Habtu, M. (2015) Qualitative study of factors associated with home deliveries and practices in Kilifi County, Kenya. International Journal of Health Sciences and Research 5(12), 369-376.

Mtumbuka, E., Maluwa, A., Pindani, A. \& Bultemeier, K. (2012) Factors influencing pregnant women to undergo HIV testing and counselling during antenatal clinic in Malawi. Journal of Research in Nursing and Midwifery 1(4), 56-61.

Muchabaiwa, L., Muzambani, D., Chigusiwa. L., Bindu, S. \& Mudavanhu, V. (2012) Determinants of maternal healthcare utilisation in Zimbabwe. International Journal of Economic Science and Applied Research 5(2), 145-162.

Mucheto, P., Chadambuka, A., Shambira, G. et al. (2011) Determinants of nondisclosure of HIV status among women attending the prevention of mother to child transmission programme, Makonde district, Zimbabwe. Pan African Medical Journal 8, 51.

Munjoma, M. W., Mhlanga, F. G., Mapingure, M. P., Kurewa, E. N., Mashavave, G. et al. (2010) The incidence of HIV among women recruited during late pregnancy and followed up for six years after childbirth in Zimbabwe. BMC Public Health 10, 668.

Murire, M. N., Campbell, C. \& Gregson, C. (2014) Community group membership and stigmatising attitudes towards people living with HIV in Eastern Zimbabwe. Journal of Community Health 39(1), 72-78

Musarandega, R., Machekano, R., Chideme, M., Muchuchuti, C., Mushavi, A., Mahomva, A. \& Guay, L. (2017) PMTCT service uptake among adolescents and adult women attending antenatal care in selected health facilities in Zimbabwe. Journal of Acquired Immune Deficiency Syndromes 75,148-155

Ngome, E. \& Odimegwu, C. (2014) The social context of adolescent women's use of modern contraceptives in Zimbabwe: a multilevel analysis. Reproductive Health Journal 11, 64

Obure, A. D., Jacobs, R., Guinness, L., Mayhew, S. \& Vassall, A. (2016) Does integration of HIV and sexual and reproductive health services improve technical efficiency in Kenya and Swaziland? An application of a two-stage semi parametric approach incorporating quality measures. Social Science \& Medicine 151, 147-156.

Ochako, R. \& Gichuh, W. (2016) Pregnancy wantedness, frequency and timing of antenatal care visit among women of childbearing age in Kenya. Reproductive Health 13(1), 51.

O'Meara, W. P., Platt, A., Naanyu, V. \& Ndege, S. (2013) Spatial autocorrelation in uptake of antenatal care and relationship to individual, household and village-level factors: results from a community-based survey of pregnant women in six districts in western Kenya. International Journal of Health Geographics 12, 55.

Renju, J., Andrew, B., Kishamawe, C., Kato, C., Changalucha, J. \& Obasi A. (2010) A process evaluation of the scale up of a youth friendly health services initiative in northern Tanzania. Journal of the International AIDS Society 13, 32-10.

Rutaremwa, G. \& Kabagenyi, A. (2016) Utilization of integrated HIV and sexual and reproductive health services among women in Uganda. BMC Health Services Research 20(16), 494.

Sahlu, I., Howe, C. J., Clark, M. A. \& Marshall, B. D. L. (2014) HIV status, knowledge of mother-to-child transmission of HIV and antenatal care use among Ethiopian women. Journal of Epidemiology and Global Health 4, 144-184.

Sama, C-B., Feteh, V. F., Tindong, M., Tanyi, J. T. \& Bihle, N. M. (2017) Prevalence of maternal HIV infection and knowledge on mother-to-child transmission of HIV and its prevention among antenatal care attendees in a rural area in northwest Cameroon. PLoS One 12, 2.

Semali, I. A., Jeremia, D., Saronga, H. \& Malamsha, D. (2014) Factors associated with HIV testing and receiving results during antenatal care in Tanzania. African Population Studies 28(2), 000-000.

Sharma, M., Barnabas, R. V. \& Celum, C. (2017) Community-based strategies to strengthen men's engagement in the HIV care cascade in sub-Saharan Africa. PLoS Medicine 14(4), 000-000.

Shetty, A. K., Chandisarewa, W., Mahomva, A. \& Mushava, A. (2010) Preventing mother-to-child transmission of HIV in urban Zimbabwe. Journal of Perinatology 30(11), 763-764.

Takarinda. K.C., Madyira, L.K., Mhangara. M., Makaza, V., Maphosa-Mutsaka, M., Rusakaniko, S et al. (2016) Factors Associated with Ever Being HIV Tested in Zimbabwe: An Extended Analysis of the Zimbabwe Demographic and Health Survey (2010- 2011). PLOS ONE, 11 (1).

Tarekegn, M. S., Lieberman, L. S. \& Giedraitis, V. (2014) Determinants of maternal health service utilisation in Ethiopia: analysis of the 2011 Ethiopian Demographic and Health Survey. BMC Pregnancy and Childbirth, 14-161

Tenthan, L., Haas, H. D. \& Egger, M. (2015) HIV testing among pregnant women who attend antenatal care in Malawi. Journal of Acquired Immune Deficiency Syndromes 69, 610-614.

Titaley, C. R., Hunter, C. L., Heywood, P. \& Dibley, M. J. (2010) Why don't some women attend antenatal and postnatal care services? A qualitative study of community members' perspectives in Garut, Sukabumi and Ciamis districts of west Java province Indonesia. BMC Pregnancy and Child Birth 10, 61.

Turan, J. M., Miller, S, Bukusi, E. A., Sanded, J. \& Cohen, C. R. (2008) HIV/AIDS and maternity care in Kenya: how fears of stigma and discrimination affect uptake and provision of labour and delivery services. AIDS Care 20(8), 938-945.

Tweya, H., Gugsa, S., Hosseinipour, M. et al. (2014) Understanding factors, outcomes and reasons for loss to follow-up among women in Option B+ PMTCT programme in Lilongwe, Malawi. Tropical Medicine \& International Health 19, $1360-1366$

Underwood, C. R. \& Schwand, H. M. (2016) Assessing girls' HIV vulnerability: evidence from Botswana, Malawi and Mozambique. Health Policy and Planning 31, 729-735

Washington, S., Owuor, K., Turan, J. M., Steinfeld, R. L., Onono, M., Shade, S. B. et al. (2015) The effect of integration of HIV care and treatment into antenatal care clinic son mother-to-child HIV transmission and maternal outcomes in Nyanza, Kenya: results from the SHAIP cluster randomized controlled trial. Journal of Acquired Immune Deficiency Syndromes 69, e16471.

WHO (2015) New HIV Testing Guidance to Help Reach the 90-90-90 Targets. URL: http://www.who.int/hiv/mediacentre/news/hiv-testing-90targets/en/ (accessed $15^{\text {th }}$ August 2017).

Woldesenbet, S., Jackson, D., Lombard, C. et al. (2015) Missed opportunities along the prevention of mother-to-child transmission services cascade in South Africa: uptake, determinants, and attributable risk. PLoS One 10, e0132425. 
Zimbabwe National Statistics Agency (ZIMSTAT) and ICF International (2012) Zimbabwe Demographic and Health Survey 2010-11. Calverton, Maryland: ZIMSTAT and ICF International Inc. Available: http://dhsprogram.com/pubs/pdf/FR254/FR254.pdf. Assessed May 2017.

ZIMBABWE NATIONAL HIV AND AIDS STRATEGIC PLAN [ZNASP II] (2011-2015) .Delivering our commitment to, Zero new HIV infections, Zero discrimination, Zero AIDS-related deaths, Republic of Zimbabwe. Web site .http://www.nac.org.zw/. Accessed August 3, 2017

Fig. 1. Conceptual framework for determinants of HIV and ANC health integrated service utilization. Adapted from Andersen (1995). 
Table 1. Description of study variables

\section{Definition}

\section{Outcome variable}

Antenatal HIV testing

\section{Individual predisposing factors}

Residence

Age group

Education

Marital status

Religion

Wanted last child

Parity
The binary variable was constructed based on if the pregnant women had been offered and she had accepted an HIV test for her last birth during ANC visits. The measure was based on a re-code of the DHS variable V840, in which a women who had been tested for HIV as part of her ANC was coded ' 1 ' and ' 0 ' otherwise.

Women's residence, coded as $1=$ rural, and $0=$ urban

Categorized into three groups: $15-24,25-34$ and $35+$ years

Educational attainment, classified into two groups: 'no education and primary' coded ' 0 ' and 'secondary and higher' coded ' 1 '

Classified into four groups: never married, currently married, widowed and divorced/separated

Religious affiliation, classified into: Catholic/Traditional, Protestant, Pentecostal, Apostolic

Three categories: wanted then, wanted later and not wanted

Total number of children ever born, classified into three groups: 1, 2 and $3+$

\section{Enabling and perceived risk/need factors}

Media exposure

Household wealth

Autonomy

Ever been tested for HIV

HIV stigma

HIV awareness

Knows someone living with HIV

Confidentiality concern
A composite summary index derived from information on frequency of reading newspapers, listening to radio and watching TV by respondents. The additive scores, ranging from $0-1$, were coded: '0' for low', '2-3' was ' 1 ' 'medium' and 4-5 was '2' 'higher'

DHS composite wealth index (Rustein et al., 2004), based on household possessions and amenities, re-classified into three groups (poor, rich and richest) based on Muchabaiwa et al. (2012)'s classification of poverty in Zimbabwe

The relevant DHS questions (mv739 to mv743b) on who makes the final decision on woman's health care elicited the categorical responses: $1=$ woman alone, $2=$ jointly and $3=$ husband alone/others

Coded as $1=$ if respondent had ever been tested for HIV; $0=$ otherwise

A composite index derived from a set of four DHS questions on HIV/AIDS stigma. The resulting score was classified as $0=$ low, $1-2=$ medium and $3-4=$ high

A composite index derived from a series of questions on knowledge of how HIV is transmitted and ways to avoid infection. The resulting index was classified into quartiles and the higher the index, the higher the knowledge.

Coded as $1=$ if respondent personally knows someone living with HIV and $0=$ otherwise

Coded $1=$ if the respondent wants HIV in the family to remain secret and $0=$ otherwise

\section{Contextual community/cluster HIV factors}

HIV prevalence

HIV awareness
Proportion of individuals in cluster who are HIV positive

Average HIV awareness in the cluster

Average HIV/AIDS stigma in the cluster 
Table 2. Percentage distribution of sample women by selected socio-demographic factors, 2005/6 and 2010/11 ZDHS

\begin{tabular}{|c|c|c|c|c|}
\hline Variable & $\%^{\mathrm{a}}$ & $n^{\mathrm{b}}$ & $\%^{\mathrm{a}}$ & $n^{\mathrm{b}}$ \\
\hline \multicolumn{5}{|l|}{ Residence } \\
\hline Urban & 31.3 & 1136 & 31.2 & 1339 \\
\hline Rural & 68.7 & 2938 & 68.8 & 3058 \\
\hline \multicolumn{5}{|c|}{ Age group } \\
\hline $15-24$ & 38.9 & 1584 & 36.2 & 1592 \\
\hline $25-29$ & 25.9 & 1054 & 29.2 & 1284 \\
\hline $30-34$ & 18.9 & 759 & 17.9 & 789 \\
\hline $35+$ & 16.6 & 677 & 16.6 & 732 \\
\hline
\end{tabular}

Marital status

\section{Never married}

Married

Widowed

Divorced/separated

Education

None/primary

Secondary/higher

Religion

Catholic/other

Protestant

Pentecostal

Apostolic

Household wealth

\section{Poor}

Rich

Richest

Wanted child

Then
Later
Not wanted

20.7

5.3

81.8

4.7

8.1

39.3

60.7

16.6

34.7

63.5

20.3

16.2

63.2

2623

20.2

16.6

1648

2426

1179

830

646

1419

2584

842

648

879

564
32.8

67.2

83.4

3.0

8.1
66.7

63.2

20.9

15.9

20.6

12.6
2921

1134

1480

2917

1107

597

799

1894

2692

965

740

340 


\begin{tabular}{|c|c|c|c|c|}
\hline \multicolumn{5}{|l|}{ Parity } \\
\hline 1 & 30.1 & 1202 & 29.5 & 1301 \\
\hline 2 & 25.6 & 1033 & 27.9 & 1225 \\
\hline $3+$ & 44.0 & 1839 & 42.6 & 1871 \\
\hline \multicolumn{5}{|c|}{ Media exposure } \\
\hline Low & 37.1 & 1567 & 31.9 & 1431 \\
\hline Medium & 29.4 & 1192 & 49.7 & 2138 \\
\hline High & 33.4 & 1315 & 18.8 & 828 \\
\hline All & 100.0 & 4074 & 100.0 & 4397 \\
\hline
\end{tabular}

${ }^{\mathrm{a}}$ Weighted; ${ }^{\mathrm{b} u n w e i g h t e d .}$ 
Table 3. Bivariate analysis of antenatal HIV testing of sample women by selected individual predisposing factors, 2005/6 and 2010/11 ZDHS

\begin{tabular}{|c|c|c|c|c|}
\hline \multirow[b]{2}{*}{ Variable } & \multicolumn{2}{|c|}{ ZDHS 2005/6 } & \multicolumn{2}{|c|}{ ZDHS 2010/11 } \\
\hline & Percentage tested ${ }^{\mathrm{a}}$ & $p$-value & Percentage tested $^{\mathrm{a}}$ & $p$-value \\
\hline Residence & & $<0.001$ & & $<0.001$ \\
\hline Urban & 40.5 & & 58.0 & \\
\hline Rural & 24.4 & & 54.9 & \\
\hline Age group & & $<0.001$ & & $<0.001$ \\
\hline $15-24$ & 34.6 & & 62.5 & \\
\hline $25-29$ & 28.3 & & 56.4 & \\
\hline $30-34$ & 27.9 & & 54.4 & \\
\hline $35+$ & 20.5 & & 42.1 & \\
\hline Marital status & & $<0.001$ & & $<0.001$ \\
\hline Never married & 27.6 & & 64.4 & \\
\hline Married & 29.9 & & 56.6 & \\
\hline Widowed & 25.2 & & 38.1 & \\
\hline Divorced/separated & 28.6 & & 49.2 & \\
\hline Education & & $<0.001$ & & $<0.001$ \\
\hline No/primary & 20.9 & & 47.0 & \\
\hline Secondary/higher & 35.0 & & 60.2 & \\
\hline Religion & & $<0.001$ & & $<0.001$ \\
\hline Catholic/other & 25.3 & & 55.5 & \\
\hline Protestant & 34.1 & & 59.5 & \\
\hline Pentecostal & 36.1 & & 55.7 & \\
\hline Apostolic & 26.8 & & 55.0 & \\
\hline Wanted child & & $<0.001$ & & $<0.001$ \\
\hline Then & 31.5 & & 55.6 & \\
\hline Later & 25.8 & & 56.2 & \\
\hline Not wanted & 25.5 & & 57.8 & \\
\hline Parity & & $<0.001$ & & $<0.001$ \\
\hline 1 & 36.9 & & 58.3 & \\
\hline 2 & 31.7 & & 58.6 & \\
\hline $3+$ & 23.0 & & 52.3 & \\
\hline
\end{tabular}


Media exposure

Low

Medium

High

Total

20.7

31.6

37.2

29.4

${ }^{\mathrm{a}}$ Weighted values.
$<0.001$

$<0.001$

49.6

59.5

44.5

55.9 
Table 4. Bivariate analysis of antenatal HIV testing of sample women by enabling and perceived risk/need factors, 2005/6 and 2010/11 ZDHS

\begin{tabular}{|c|c|c|c|c|}
\hline \multirow[b]{2}{*}{ Variable } & \multicolumn{2}{|c|}{ ZDHS 2005/6 } & \multicolumn{2}{|c|}{ ZDHS 2010/1 } \\
\hline & Percentage tested ${ }^{\mathrm{a}}$ & $p$-value & Percentage tested ${ }^{\mathrm{a}}$ & $p$-value \\
\hline HIV awareness & & $<0.001$ & & $<0.001$ \\
\hline Low & 26.1 & & 48.0 & \\
\hline Medium & 34.8 & & 59.8 & \\
\hline High & 26.7 & & 59.5 & \\
\hline Knows someone living with HIV & & $<0.001$ & & $<0.001$ \\
\hline Yes & 26.9 & & 55.9 & \\
\hline No & 33.5 & & 58.2 & \\
\hline HIV stigma & & $<0.001$ & & $<0.001$ \\
\hline Low & 26.2 & & 57.1 & \\
\hline Medium & 32.0 & & 55.9 & \\
\hline High & 28.8 & & 51.3 & \\
\hline Household wealth & & $<0.001$ & & $<0.001$ \\
\hline Poor & 23.0 & & 53.1 & \\
\hline Rich & 37.0 & & 59.5 & \\
\hline Richest & 41.8 & & 61.0 & \\
\hline Accessibility problems & & $<0.001$ & & $<0.001$ \\
\hline No & 37.1 & & 61.5 & \\
\hline Yes & 26.7 & & 52.7 & \\
\hline
\end{tabular}

${ }^{\mathrm{a} W e i g h t e d ~ v a l u e s . ~}$ 
Table 5. Average odds ratios (AOR) of antenatal HIV testing of sample women from multilevel logistic regression models

\begin{tabular}{llll}
\hline Variable & Model 1 & Model 2 & Model 3 \\
\hline Predisposing factors & & & \\
2010/11 ZDHS (Ref.: 2005/6) & $3.77(3.32-4.29)^{*}$ & $3.10(2.66-3.62)^{*}$ & $1.87(1.58-2.21)^{*}$ \\
Urban residence (Ref.: rural) & $0.52(0.43-0.63)^{*}$ & $0.83(0.60-1.13)$ & $0.97(0.68-1.39)$ \\
Age group (Ref.: 15-24) & $0.71(0.62-0.81)^{*}$ & & $0.68(0.60-0.75)^{*}$ \\
$25-29$ & $0.69(0.56-0.7)^{*}$ & $0.70(0.61-0.80)^{*}$ & $0.65(0.55-0.77)^{*}$ \\
$20-34$ & $0.43(0.36-0.52)^{*}$ & $0.65(0.55-0.78)^{*}$ & $0.42(0.35-0.51)^{*}$ \\
$35+$ & & $0.35-0.52)^{*}$ &
\end{tabular}

Marital status (Ref.: never married)

\begin{tabular}{|c|c|c|c|}
\hline Married & $1.10(0.85-1.32)$ & $1.03(0.83-1.29)$ & $1.02(0.82-1.28)$ \\
\hline Widowed & $0.75(0.54-1.06)$ & $0.73(0.52-1.02)$ & $0.47(0.47-0.93)^{*}$ \\
\hline Divorced/separated & $0.74(0.57-0.96)^{*}$ & $0.73(0.56-0.95)^{*}$ & $0.73(0.56-0.95)^{*}$ \\
\hline Secondary education (Ref.: primary) & $1.43(1.28-1.61)^{*}$ & $1.38(1.23-1.55)^{*}$ & $1.32(1.17-1.48)^{*}$ \\
\hline \multicolumn{4}{|l|}{ Religion (Ref.: Catholic/other) } \\
\hline Protestant & $1.17(0.96-1.43)$ & $1.13(0.90-1.42)$ & $1.13(0.90-1.41)$ \\
\hline Pentecostal & $1.13(0.91-1.38)$ & $1.03(0.81-1.30)$ & $1.01(0.80-1.28)$ \\
\hline Apostolic & $1.15(0.97-1.36)$ & $1.23(1.02-1.49)^{*}$ & $1.23(1.02-1.49)^{*}$ \\
\hline \multicolumn{4}{|l|}{ Wanted child (Ref.: now) } \\
\hline Later & $0.88(0.75-1.04)$ & $0.81(0.68-0.98)^{*}$ & $0.81(0.67-0.98)^{*}$ \\
\hline Not wanted & $1.15(0.93-1.43)$ & $1.16(0.90-1.49)$ & $1.16(0.90-1.49)$ \\
\hline \multicolumn{4}{|l|}{ Parity (Ref.: 0-1) } \\
\hline 2 & $0.72(0.58-0.90)^{*}$ & $0.90(0.73-1.11)$ & $0.90(0.73-1.11)$ \\
\hline $3+$ & $0.88(0.75-1.04)$ & $0.78(0.61-0.99)^{*}$ & $0.78(0.61-1.00)$ \\
\hline
\end{tabular}

\section{Enabling/need factors}

Wealth (Ref.: poor)

Rich

$1.45(1.11-1.91)^{*}$

$1.35(1.02-1.78)^{*}$

Richest

$1.67(1.16-2.41)^{*}$

$1.53(1.05-2.24)^{*}$

Media exposure (Ref.: low)

Medium

$1.35(1.12-1.63)^{*}$

$1.34(1.10-1.64)^{*}$

High

$1.30(1.05-1.61)^{*}$

$1.28(1.03-1.60)^{*}$

Access problem (Ref.: no)

$0.86(0.75-0.98)^{*} \quad 0.85(0.75-0.97)^{*}$ 
HIV stigma (Ref.: low)

Medium

High

Knows someone with AIDS (Ref.

no)

\section{Contextual factors}

Community HIV awareness

HIV stigma in community

\section{Community random effects}

\begin{tabular}{llll} 
Variance (SE) & $0.266(0.036)^{*}$ & $0.25(0.035)^{*}$ & $0.241(0.034)^{*}$ \\
$(\mathrm{VPC})=\mathrm{ICC}(\%)$ & 8 & 7 & 7 \\
$(\mathrm{PCV})(\%)$ & 12.5 & 0 & 0 \\
\hline
\end{tabular}

95\% confidence intervals in parentheses.

${ }^{*} p<0.05$.

$\begin{array}{ll}1.41(1.14-1.73)^{*} & 1.36(1.10-1.68)^{*} \\ 1.11(0.87-1.42) & 1.07(0.3-1.38) \\ 1.23(1.02-1.49)^{*} & 1.24(1.02-1.51)^{*}\end{array}$

$2.08(1.05-4.11)^{*}$

$0.68(0.52-0.87)^{*}$ 